\title{
Pneumatosis intestinalis as a complication of acute diarrhoea in children
}

\author{
Sabina Więcek' ${ }^{1}$ Urszula Grzybowska-Chlebowczyk' , Zbigniew Olczak², Katarzyna Gruszczyńska³, \\ Klaudia Korecka ${ }^{4}$, Tomasz Koszutski ${ }^{4}$ \\ 'Department of Paediatrics, School of Medicine in Katowice, Medical University of Silesia, Katowice, Poland \\ ${ }^{2}$ Division of Diagnostic Imaging and Interventional Radiology, Upper Silesian Medical Centre, Katowice, Poland \\ ${ }^{3}$ Department of Diagnostic Imaging, School of Medicine in Katowice, Medical University of Silesia, Katowice, Poland \\ ${ }^{4}$ Department of Child Surgery, School of Medicine in Katowice, Medical University of Silesia, Katowice, Poland
}

\section{ABSTRACT}

Introduction: Pneumatosis of the intestine is often mentioned in the course of necrotising enterocolitis (NEC) in premature infants.

Aim of the study: The aim of the study was to assess the course of pneumatosis intestinalis in acute diarrhoea and to analyse the factors in its development.

Material and methods: The analysis included 10 children aged from three months to six years. All children underwent abdominal ultrasound examination, which revealed pneumatosis of the intestine. The analysis included pregnancy and delivery history, clinical symptoms, and the results of laboratory tests.

Results: Bloody diarrhoea dominated the clinical picture in 9/10 patients (90\%). Abnormal pregnancy/delivery history was reported in six children. NEC was concluded in 3/10 children. Infections with rotavirus were reported win 7/10 patients, Klebsiella pneumoniae were cultured in the stools of 4/10 children. Food allergy was observed in 5/10 patients and immunoglobulin deficiency in three children.

Conclusions: This study describes some cases of pneumatosis intestinalis as a complication following acute diarrhoea, as observed in children below the age of 12 months. Abnormalities in pregnancy/delivery history, low body mass, and co-occurrence of food allergy were the risk factors.

\section{KEY WORDS:}

pneumatosis intestinalis, children, bloody diarrhoea.

\section{INTRODUCTION}

Pneumatosis intestinalis is defined as the presence of gas within the wall of the gastrointestinal tract. The gas may originate from the gastrointestinal lumen, bacterial production, or from the lungs. It was originally described on plain abdominal radiographs, but nowadays in ultrasonography. The lesions usually affect the small intestine; however, cases of pneumatosis affecting the large intestine have been reported.

The pathogenesis remains uncertain but is believed to be the result of multiple contributing factors. The bacterial theory is as follows: the gas is produced by gas-forming bacteria that enter the mucosal barrier through mucosal rents or increased mucosal permeability and produce the gas within the intestine wall. Pneumatosis intestinalis is

\section{CORRESPONDENCE ADDRESS:}

Sabina Więcek, Department of Paediatrics, School of Medicine in Katowice, Medical University of Silesia, 16 Medyków St., 40-752 Katowice, Poland, e-mail: sabinawk@wp.pl 
most often described in the course of necrotising enterocolitis (NEC) in premature infants and during graft-versus-host-reactions. Factors promoting the development of pneumatosis intestinalis also include heart defects, immune disorders, and ischaemic and autoimmune processes. Symptoms can include diarrhoea, constipation, mucus per rectum, bleeding, flatus, abdominal pain, and rarely faecal incontinence. There are limited studies concerning this complication as pneumatosis intestinalis in the course of acute diarrhoea. The clinical course can vary, from benign diseases to abdominal sepsis and death [1-5].

The aim of the study was to evaluate the course of, and analyse the factors affecting, the occurrence of pneumatosis intestinalis in the course of acute diarrhoea in children.

\section{MATERIAL AND METHODS}

We analysed 10 children, aged from three months to six years (mean age eight months), five girls and five boys, treated in the Department of Paediatrics of the Medical University of Silesia in Katowice in the years 2011-2015 for acute diarrhoea with accompanying pneumatosis intestinalis (Table 1).

Abdominal ultrasound, in which we found pneumatosis of the intestine, was performed in all children by a radiologist with more than five years' experience in paediatric radiology. The ultrasound system Logiq Q7 (GE Medical Systems) was used, a 3.5-5-MHz convex and $9-\mathrm{MHz}$ linear transducer was used for the liver imaging, and an 11-MHz linear transducer was used for the intestinal wall imaging. Gestational and labour history, clinical signs, and the results of the laboratory tests were evaluated.

The research work has been reported in line with the process criteria [6].

\section{RESULTS}

The diagnosis of pneumatosis intestinalis was given based on the abdominal ultrasound - the presence of oxygen within the intestinal walls, and in 8/10 (80\%) also in the portal vein (Fig. 1, Fig. 2). Pneumatosis intestinalis was diagnosed in eight infants and two older children (aged three and six years).

Positive gestational and labour histories (prematurity, dyspnoea) were observed in six patients with pneumatosis intestinalis $-60 \%$. Children with abnormal pregnancy/delivery histories were concluded to have had a more acute course of diarrhoea and required longer hospitalisation and longer parenteral nutrition. NEC in the history of newborns was found in $3 / 10$ children.

Diarrhoea with blood predominated in the clinical picture of $9 / 10$ of the examined children (90\%). Pneumonia was additionally concluded in one patient.
Rotavirus infection was diagnosed in 7/10 patients. Klebsiella pneumoniae was cultured in the stools of 4/10 children and Citrobacter freundii was cultured in the stools of one patient.

Elevated indicators of inflammation (C-reactive protein and procalcitonin) were observed in 5/10 patients (50\%). Heightened activity of aminotransferase and/or guanosine triphosphate (GTP) was reported in four children. Anaemia was observed in 5/10 children (50\%) (Hb - 9.5-10.5 g). Higher concentrations of lactic acid in the blood serum were observed in the children with a more severe course of the condition.

IgE-dependent food allergy (to cow`s milk protein and egg protein in the older group) was diagnosed in five children and immunoglobulin deficiency in three children (isolated deficiency in A immunoglobulins in the case of one child and deficiency in type A and G in two children). Five children had a history of co-existing haemodynamically significant heart conditions (atrial septal defect - ASD, ventricular septal defect - VSD, foramen ovale apertum - FoA, interatrial septum - IAS, and pulmonary hypertension).

The treatment of all the patients involved antibiotic therapy and total parenteral nutrition, which improved the general condition and led to the withdrawal of the symptoms and pneumatosis of the intestine in ultrasound.

\section{DISCUSSION}

The frequency of pneumatosis in the intestinal wall is estimated at $0.03 \%$ of the general population. In recent years and thanks to the more frequent use of abdominal ultrasonography and computer tomography (in adults) there has been an increase in the number of diagnosed cases of pneumatosis. The causes of pneumatosis intestinalis are traditionally divided into two categories: benign conditions (such as asthma, scleroderma, pyloric stenosis) and life-threatening conditions (such as bowel obstruction, necrotic enterocolitis, bowel ischaemia, cancer, trauma, and drugs). Pneumatosis of the intestinal walls in infants is in most cases associated with prematurity and perinatal hypoxia - and is diagnosed within the first few months of life. The aetiopathogenesis of the condition is complex and not fully understood - it is believed that ischaemic, pro-inflammatory, and immunological factors play a role. Cases when air bubbles in the walls of the gastrointestinal tract were present since birth - about $10-15 \%$ of all patients with pneumatosis of the gastrointestinal wall - are known. So, the pathomechanism seems to be even more complex [7-11].

In 2015 He brought forward a theory in which he mentioned the influence of aerophagy, when air gathers in the lumen of the stomach and the initial parts of intestinal loops. Gastric juice probably contributes to the air penetration of the gastrointestinal walls [12]. However, pneumatosis intestinalis may also be the result of numerous non-ischaemic 


\begin{tabular}{|c|c|c|c|c|c|}
\hline 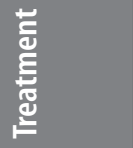 & 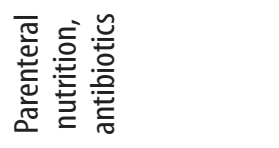 & 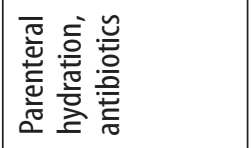 & 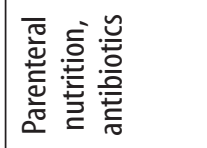 & 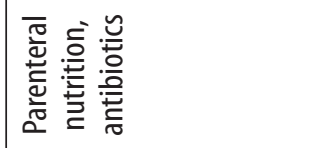 & 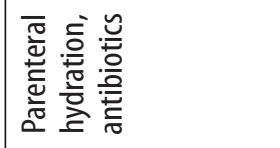 \\
\hline 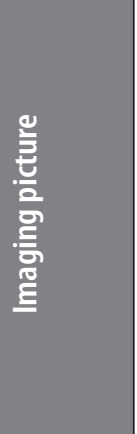 & 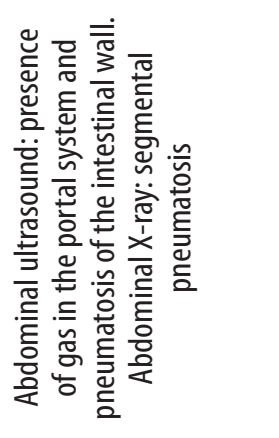 & 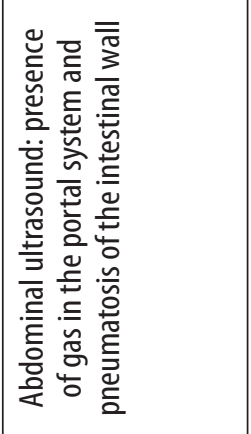 & 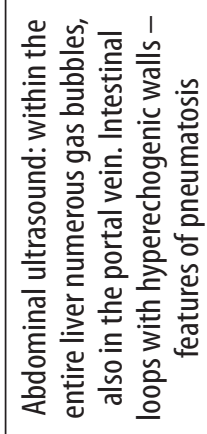 & 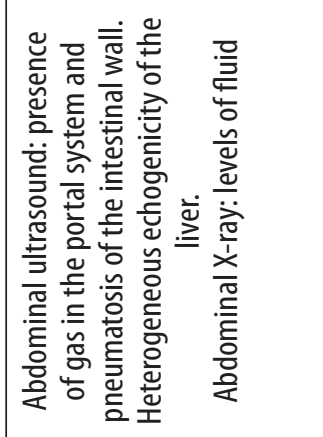 & 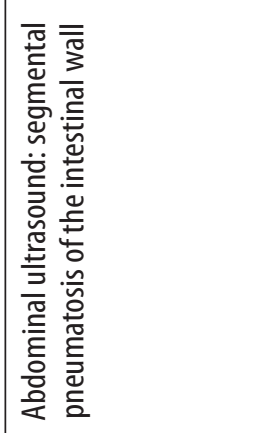 \\
\hline 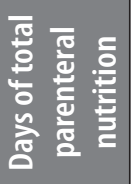 & 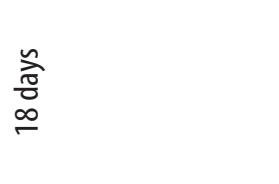 & I & 愛 & 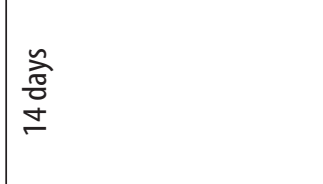 & 1 \\
\hline 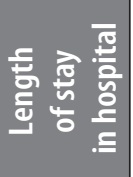 & 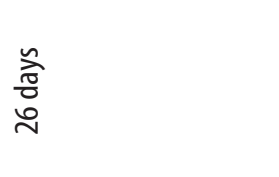 & 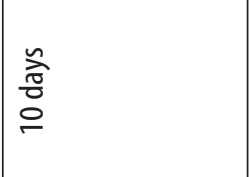 & $\begin{array}{l}\tilde{\widehat{\widehat{A}}} \\
\stackrel{0}{0} \\
0\end{array}$ & 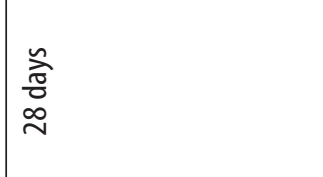 & 縞 \\
\hline 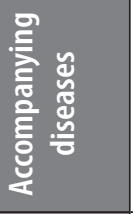 & 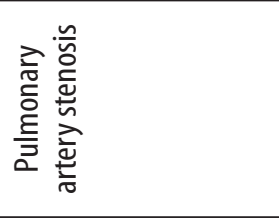 & 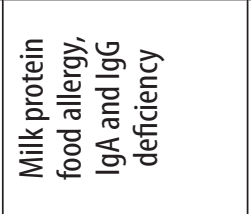 & 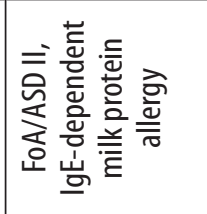 & 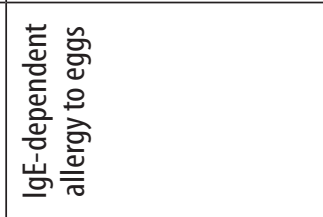 & 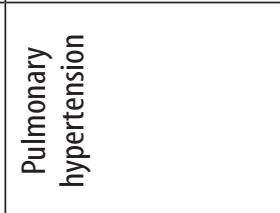 \\
\hline 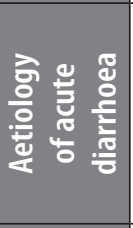 & 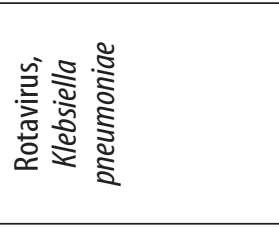 & 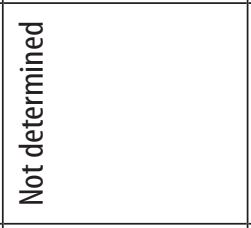 & 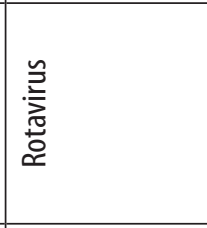 & 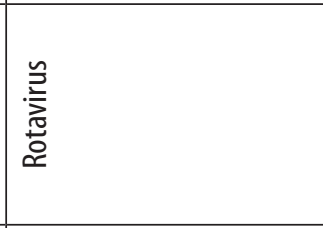 & 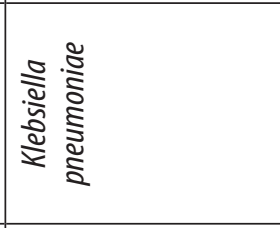 \\
\hline 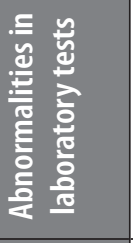 & 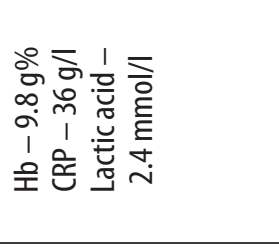 & 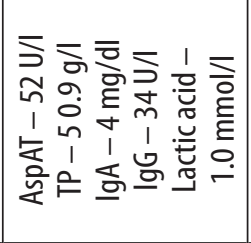 & 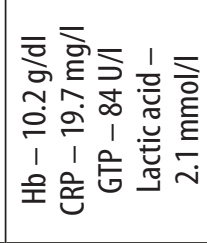 & 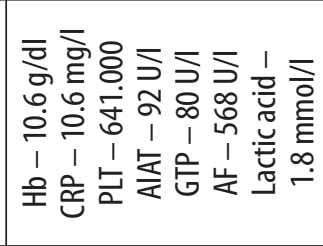 & 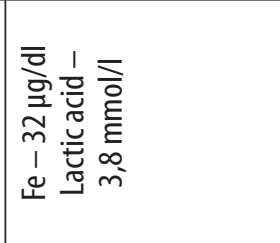 \\
\hline 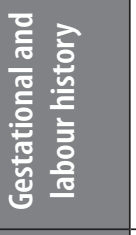 & 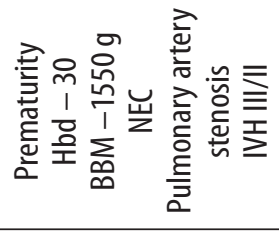 & 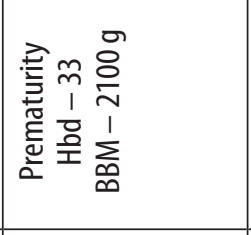 & 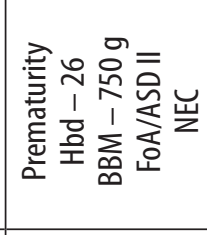 & 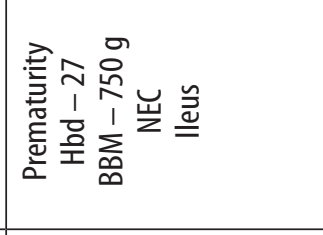 & 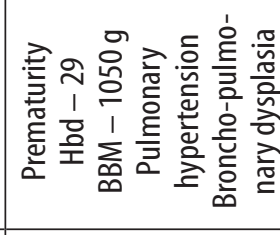 \\
\hline 를 & 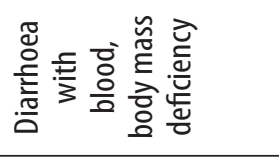 & 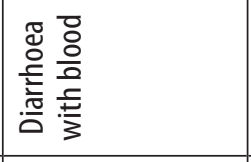 & 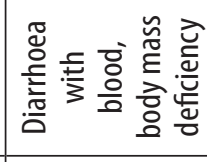 & 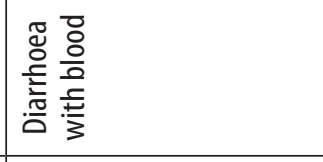 & 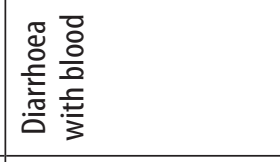 \\
\hline ঐ & 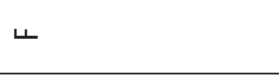 & $\Sigma$ & $\Sigma$ & ப & $\Sigma$ \\
\hline ğ & $\stackrel{\sim}{m}$ & $\stackrel{\curvearrowright}{\lessgtr}$ & $\frac{2}{6}$ & $\frac{2}{6}$ & $\stackrel{2}{\gtrless}$ \\
\hline 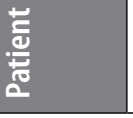 & $\stackrel{亡}{亡}$ & ì & $\begin{array}{l}\dot{0} \\
\dot{m}\end{array}$ & $\begin{array}{l}\dot{\sim} \\
\dot{+}\end{array}$ & 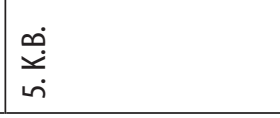 \\
\hline
\end{tabular}




\begin{tabular}{|c|c|c|c|c|c|}
\hline 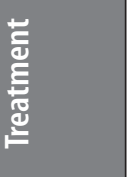 & 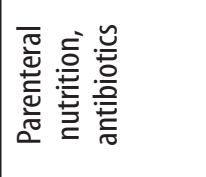 & 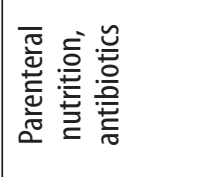 & 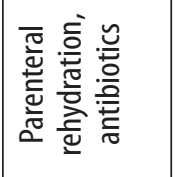 & 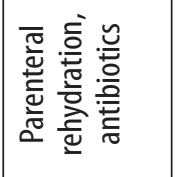 & 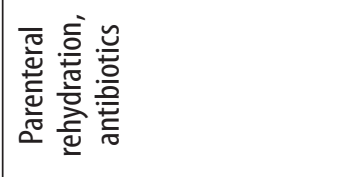 \\
\hline 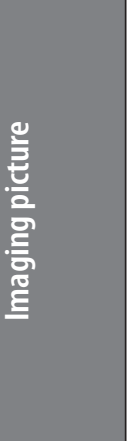 & 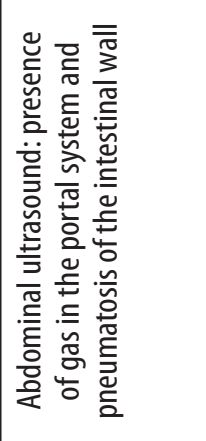 & 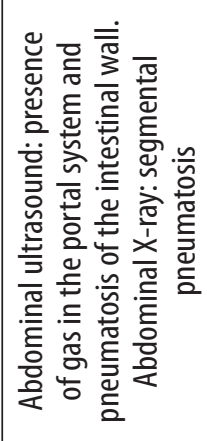 & 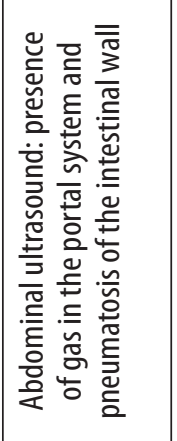 & 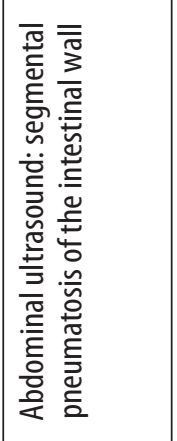 & 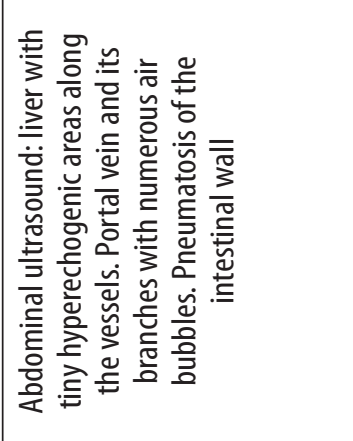 \\
\hline 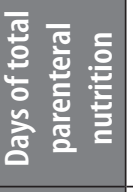 & 䒽 & 䋨 & 1 & 1 & I \\
\hline 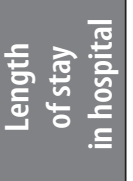 & 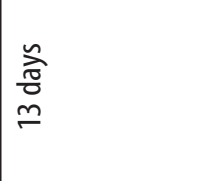 & 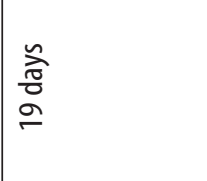 & 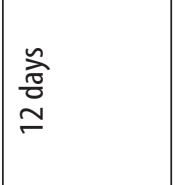 & $\begin{array}{l}\text { ầ } \\
\text { त् } \\
\infty\end{array}$ & 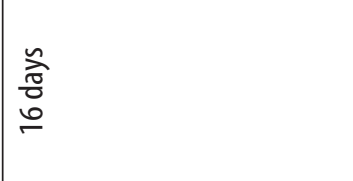 \\
\hline 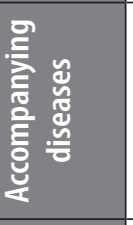 & 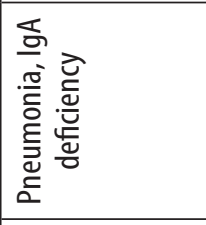 & 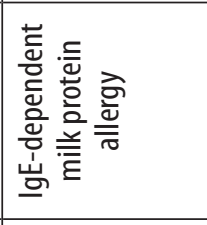 & 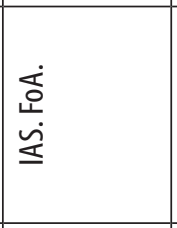 & 1 & 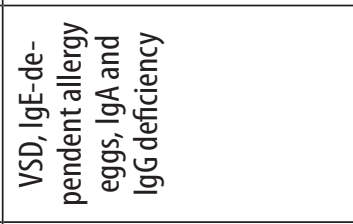 \\
\hline 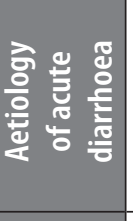 & 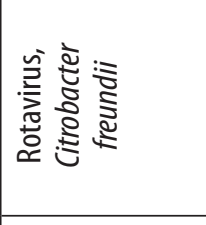 & 号 & 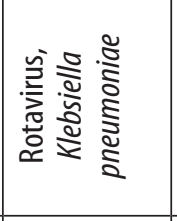 & 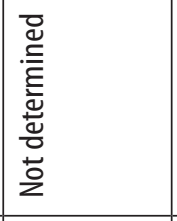 & 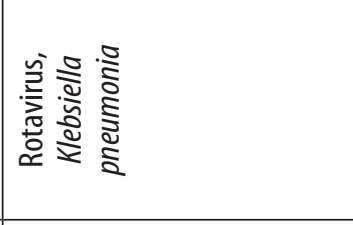 \\
\hline 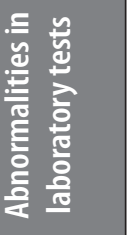 & 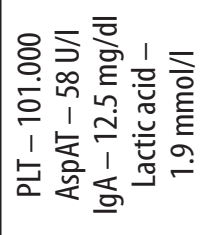 & 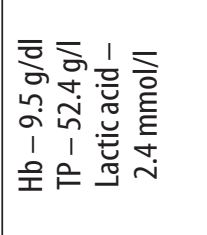 & 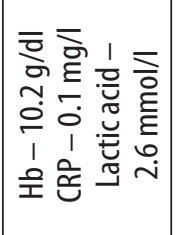 & 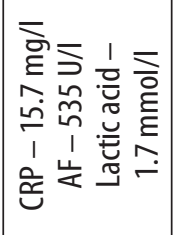 & 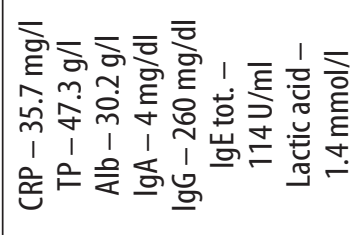 \\
\hline 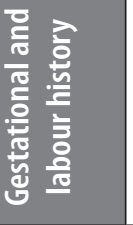 & 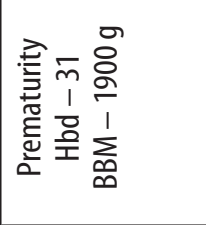 & । & 1 & 1 & जิ \\
\hline 氖 & 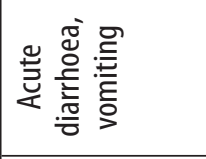 & 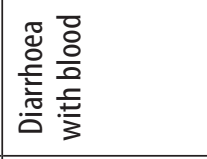 & 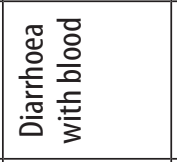 & 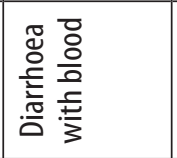 & 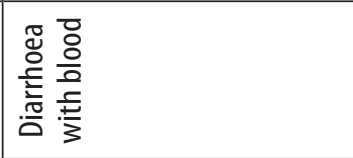 \\
\hline ঐ & 4 & 4 & $\Sigma$ & $\Sigma$ & 4 \\
\hline gั & 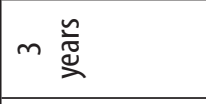 & $\stackrel{\sim}{\lambda}$ & $\stackrel{\sim}{m}$ & $\underset{0}{2}$ & 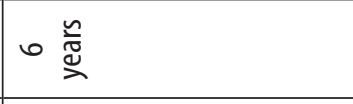 \\
\hline 莒 & స్ & 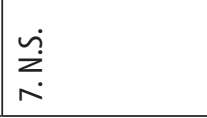 & $\stackrel{N}{\stackrel{N}{\infty}}$ & $\sum_{\substack{0 \\
\vdots}}^{3}$ & \\
\hline
\end{tabular}


and non-obstructive conditions, most of which are apparently not associated with unfavourable outcomes.

Out of 2600 patients hospitalised in the Department of Paediatrics of the Medical University of Silesia in Katowice from 2011 to 2015, pneumatosis intestinalis was diagnosed in 10 children $(0.38 \%)$. Our patients were from three months to six years old with the average age of eight months. 6/10 children had abnormal pregnancy/ delivery history, including prematurity, perinatal hypoxia and/or intrauterine infections, which may have been the factors contributing to the condition because hypoxia is known to affect the development of pneumatosis. Due to the history of NEC in three patients, it seems that the re-occurrence of the features of pneumatosis is linked to the previous abnormalities.

Haemodynamically significant myocardial diseases (VSD, ASD, FoA, IAS, pulmonary stenosis, and pulmonary hypertension) were diagnosed in 5/10 (50\%) of the children, which, due to hypoxic and ischaemic processes, may additionally affect intestinal pneumatosis. The following ischaemic factors are also considered in the pathomechanism of NEC: perinatal hypoxia, ischaemia, congenital heart disease, anaemia, and polycythaemia, as well as factors contributing to an infection, prematurity, and abnormal pregnancy-delivery history. There are studies describing frequent occurrences of intestinal pneumatosis in people with auto-immune diseases such as lupus variants, dermatomyositis, polyarteritis nodosa, scleroderma, and celiac disease [10, 12-15]. We did not report any such diseases among our patients; however, they are regularly consulted in the gastroenterology department. The question remains: does a diet affect intestinal pneumatosis? The patients in our study mostly followed diets typical for their age $-6 / 10$ were fed naturally over the age of six months. IgE-dependent food allergy to milk protein and/or egg protein was concluded in 5/10 patients. Inflammatory and immunological reactions that occur during allergy processes may indirectly affect pneumatosis intestinalis. Also, viral, bacterial, and/or fungal infections (HIV, cytomegalovirus, rotaviruses adenoviruses, smallpox-shingles, candida albicans, and mycobacterium tuberculosis) seem to be additional factors activating pneumatosis of the gastrointestinal tract. Infection with rotavirus was reported in 7/10 (70\%) patients with acute diarrhoea. Klebsiella pneumoniae was cultured in the stools of $4 / 10$ children (40\%), and Citrobacter freundii in one patient. $3 / 10$ children had viral (rotavirus) and bacterial infections (Klebsiella pneumoniae). Deficiencies in immunoglobulins $A$ and/or $G$ were shown in three children. Immunoglobulin A as a mucosal immunoglobulin plays a protective role while inhibiting the penetration of food allergens and thus suppressing the inflammatory processes. Patients treated with immunosuppressants or during chemotherapy are a group at risk of developing pneumatosis intestinalis [13, 14]. The subject literature describes cases of pneumatosis intestinalis in the course of viral and bacterial infections

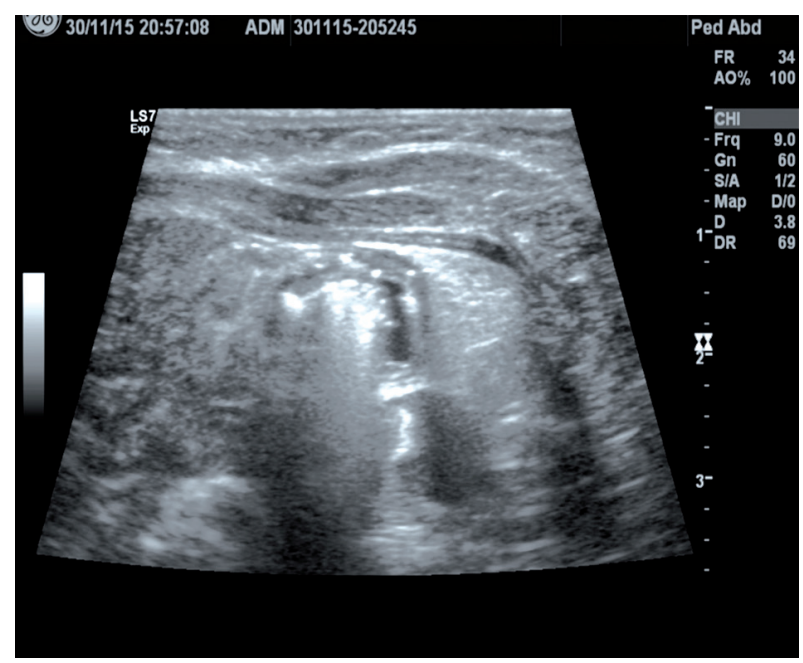

FIGURE 1. Ultrasound image of pneumatosis of the intestinal wall
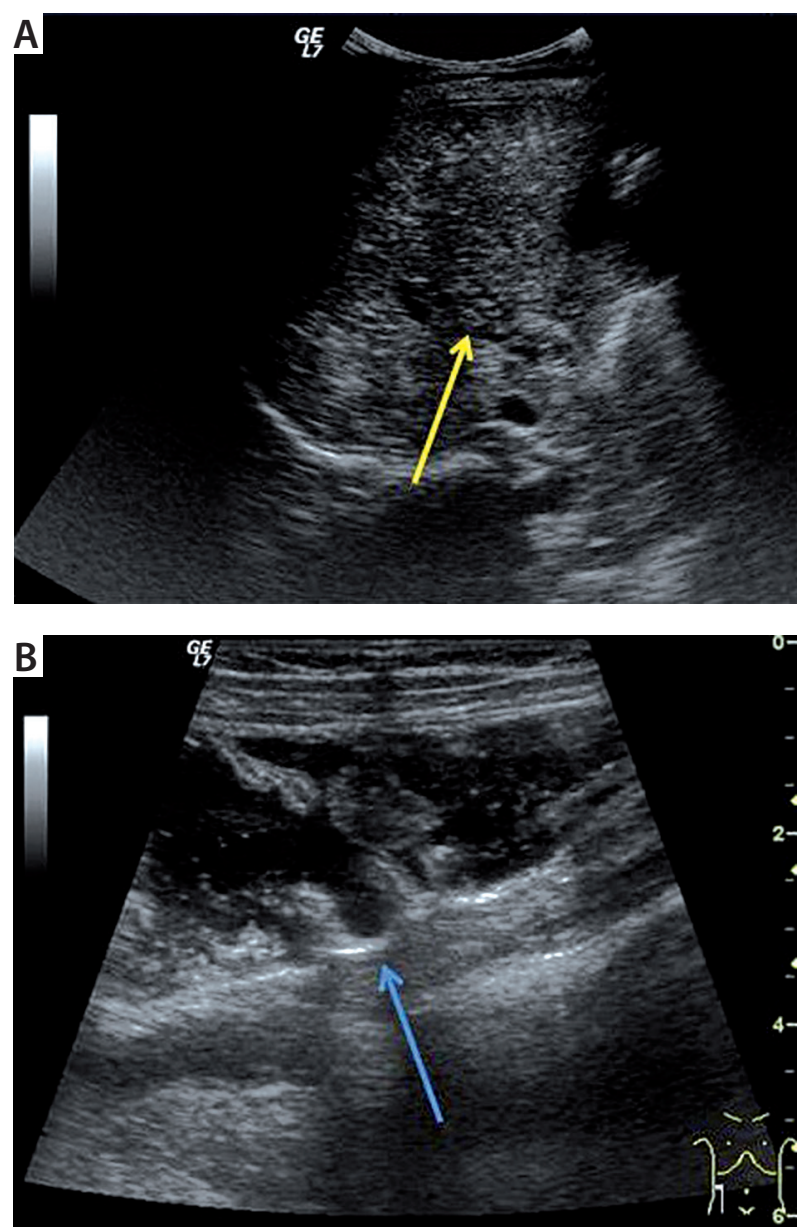

FIGURE 2. A) A substantial number of gas bubbles in the portal vein (the yellow arrow) and its branches. B) Pneumatosis of the walls of the cecum and the ascending colon (the blue arrow)

(with norovirus, cytomegalovirus, or rotavirus) in patients after organ transplant [15-17].

Medications, including corticosteroids, cytotoxic agents, lactulose, glucosidase inhibitor, and chloral hydrate are among the factors triggering pneumatosis intestinalis. At the time of the occurrence of pneumatosis 
intestinalis our patients were not being treated with the above medications. However, 6/10 (60\%) patients had very complicated pregnancy/delivery histories and required complex pharmacotherapy during the neonatal period, which may have affected the functions of the gastrointestinal tract [18-20].

The presence of pneumatosis intestinalis often leads physicians to diagnose a serious disease. Depending on the study, the clinical picture may be dominated by the symptoms of acute abdomen, abdominal bleeding and diarrhoea. Bloody diarrhoea dominated in our patients - 9/10 (90\%). Also, other authors claimed that bleeding from the gastrointestinal tract is the most common symptom of pneumatosis intestinalis - mainly manifested as bloody stools $[7,18]$.

An increase in the number of diagnosed pneumatoses of the walls of the gastrointestinal tract may be linked to the lowering mortality rate among children with severely abnormal pregnancy/delivery histories $[11,19]$.

Increased visualisation of pneumatosis intestinalis is also connected with technical improvements in gastrointestinal tract imaging techniques, mainly ultrasound (US) [19-22]. Nowadays, the intestine is routinely visualised in US with the use of high-frequency transducers $(5-12 \mathrm{~Hz})$, graded-compression techniques, and colour Doppler ultrasound. Such techniques allow for the visualisation of five separate layers of the small bowel wall. In pneumatosis intestinalis US depicts thickening of the small bowel walls above $3 \mathrm{~mm}$, the obscuring of normal small bowel wall layers, and the presence of gas bubbles within the intestinal wall. They appear as small echoic structures with acoustic shadowing within the upper (nondependent) intestinal wall [21]. The presence of gas in the portal vein is seen as multiple fast-moving echoic bubbles. US of the liver shows multiple, punctate echogenic foci in the liver - gas bubbles in small branches of the portal vein. Colour Doppler US recognises small bowel wall hypervascularisation, and increased flow velocity within the celiac and mesenteric arteries [22, 23]. Gas bubbles in the portal vein are seen as multiple bidirectional artefacts during normal portal flow in colour Doppler US. The lack of flow within the intestinal wall means wall necrosis; the pneumoperitoneum, due to intestinal perforation, is seen in US as hyperechoic foci with acoustic shadowing above the intestinal loops and the anterior surface of the liver, and below the abdominal wall. Additionally, abdominal abscesses and ascites could be detected. These findings are connected with an unfavourable outcome [22].

Whether the presence of portomesenteric venous gas as a single sign can predict the severity of ischaemia remains a controversial issue. Several studies reported a higher mortality rate with the combination of pneumatosis and portal venous gas [24]. Although plain abdominal X-ray is a traditional method of the diagnosis of pneumatosis intestinalis, we avoid it, if possible. The sign of pneumatosis in X-ray and computed tomography (CT) is the presence of gas within the lower intestinal wall, and the presence of gas in portal vein branches in the liver. CT may be useful when diagnosing pneumatosis intestinalis in adults due to frequent obesity and the restricted penetration of US waves. In children CT is connected with high $\mathrm{X}$-ray irradiation, and in small children it requires general anaesthesia, so generally CT is not recommended in children [22, 24-26]. In a benign course of the disease, the presence of intestinal and portal pneumatosis is intermitted, so US is the method of choice in recognising it [26].

The treatment of pneumatosis intestinalis remains controversial. Any surgical intervention should be limited to cases of proven perforation and/or necrosis of the bowel wall. The treatment involves wide-spectrum antibiotic therapy and parenteral nutrition and rehydration with the balancing of the electrolyte and acid-alkaline systems. There are also data confirming the efficiency of octreotide and hyperbaric oxygen [27-31]. None of our patients required surgery. Tahiri, however, claims that more than $50 \%$ of patients with secondary pneumatosis intestinalis require surgical intervention. It is possible that such a high percentage is related to the older age of the patients [19]. Factors that may lead to a negative prognosis include hypotension or vasopressor use, peritonitis, concentration of lactic acid $>2 \mathrm{mmol} / \mathrm{l}$, acute kidney failure, and mechanical ventilation. None of the above factors were reported in our patients, except for three patients with slightly elevated levels of lactic acid in the blood serum.

Due to the severe courses of the disease in our patients, the treatment involved antibiotic therapy, parenteral hydration, and in the some cases also complete parenteral nutrition. All the patients remain under the care of the gastroenterology department.

\section{CONCLUSIONS}

We present rare cases of a complication of acute diarrhoea - intestinal pneumatosis - which we observed most often in the youngest children (age below six years). The risk factors included positive gestational and labour history, low birth weight, cardiological problems, rhinovirus infection, and coexisting food allergy. Abdominal ultrasound should be performed in children with a severe course of bloody diarrhoea.

\section{DISCLOSURE}

The authors declare no conflict of interest.

\section{REFERENCES}

1. Slesser A, Patel P, Das S, et al. A rare case of segmental small bowel pneumatosis intestinalis: A case report. Int J Surg Case Rep 2011; 2: 185-187.

2. Snape J, Hulman G, Reddy P, Panto P. Pneumatosis coli: an uncommon but treatable cause of fecal incontinence. Int J Clin Pract 1998; 52: 501-503. 
3. Chavarri-Guerra Y, Jose Plata J, Cabrebere-Alexandrova T, Gamboa-Dominquez A. Pneumatosis intestinalis. Report of 3 cases. Rev Gastroenterol Mex 2000; 65: 166-170.

4. Fukumori D, Sasaki T, Matsumoto H, et al. Necrotizing enteritis with hepatic portal venous gas and pneumatosis intestinalis: report of a case. Eur J Gastroenterol Hepatol 2003; 15: 201.

5. Itazaki $\mathrm{Y}$, Tsujimoto $\mathrm{H}$, Ito $\mathrm{N}$, et al. Pneumatosis intestinalis with obstructing intussusceptions: a case report and literature review. World J Gastrointest 2016; 8: 173-178.

6. Agha RA, Fowler AJ, Rammohan S, et al. The PROCESS Statement: Preferred Reporting of Case Series in Surgery. Int J Surg 2016; 36 (Pt A): 319-323.

7. Burgazli K, Mericliler M, Greiner H, et al. Pneumatosis intestinalis: a rare cause of lower gastrointestinal bleeding. Eur Rev Med Pharmacol Sci 2013; 17: 2293-2294.

8. Dovrish Z, Amson Y, Amital H, Zissin R. Pneumatosis intestinalis presenting in autoimmine disease: a report of three patients. Ann N Y Acad Sci 2009; 1173: 199-202.

9. Guha P, Handu A, Kanojia R, Rao K. Pneumatosis intestinalis and portal venous gas resulting from acute diarrheal illness: clinical picture in an infant. J Pediatr Surg 2011; 46: 271-273.

10. Khalil P, Huber-Wagner S, Ladumer R, et al. Natural history, clinical pattern and surgical considerations of pneumatosis intestinalis. Eur J Med Res 2009; 14: 231-239.

11. West K, Rescorla F, Grosfeld J, Vane D. Pneumatosis intestinalis in children beyond the neonatal period. J Pediatr Surg 1989; 24: 818-822.

12. He T, Xu C, Ji Y, et al. Idiopathic neonatal pneumoperitoneum with favorable outcome: a case report and review. World J Gastroenterol 2015; 21: 6417-6421.

13. Kyrieleis H, Wilsterman M, Severijnen R, et al. Pneumatosis intestinalis in 9 children with an oncologic disease. Ned Tijdschr Geneeskd 2005; 149: 647-652.

14. Li S, Traubici J, Ethier M, et al. Pneumatosis intestinalis in children with acute lymphoblastic leukemia and acute myeloid leukemia. Support Care Cancer 2012; 20: 343-347.

15. Wu L, Yang Y, Dou Y, Liu Q. A systematic analysis of pneumatosis cystoids intestinalis. World J Gastroenterol 2013; 19: 4973-4978.

16. Ye X, Van J, Munoz F, et al. Noroviruses as a cause of diarrhea in immunocompromised pediatric hematopoetic stem cell and solid organ transplant recipients. Am J Transplant 2015; 15: 1874-1881.

17. Kim JM, Park Y, Joh JW, et al. Pneumatosis intestinalis after adult liver transplantation. J Korean Surg Soc 2011; 80 (Suppl 1): 47-50.

18. Shaukaut A, Obideen K, Klapproth J. Pneumatosis intestinalis: a rare cause of diarrhea and hematochezia. Clin Gastroenterol Hepatol 2006; 4: 25-28.

19. Tahiri M, Levy J, Alzaid S, Anderson D. An approach to pneumatosis intestinalis: factors affecting your management. Int J Surg Case Rep 2015; 6C: 133-137.

20. Bausch D, Hopt U, Obermaier R. Of what relevance is the diagnosis of pneumatosis intestinalis and portal gas for the surgeon? Zentralbi Chir 2009; 134: 203-208.

21. Epelman M, Daneman A, Navarro OM, et al. Necrotising enterocolitis: review of the state-of-the-art imaging findings with pathologic correlation. Radiographics 2007; 27: 285-305.

22. Silva CT, DanemanA, Navarro OM, et al. Correlation of sonographic findings and outcome in necrotizing enterocolitis. Pediatr Radiology 2007; 37: 274-282.

23. Kamali K, Hosseini SR, Ardakani SM, Farnodi MR. Complementory Value of Sonography in Early Evaluation of Necrotizing Enterocolitis. Pol J Radiol 2015; 80: 317-323.

24. Wang J, Furian A, Kaya D, et al. Pneumatosis intestinalis versus pseudo-pneumatosis: review of CT findings and differentiation. Insights Imaging 2011; 2: 85-92.
25. Blair H, Baker R, Albazaz R. Pneumatosis intestinalis an increasingly common radiological finding, bening or life-threatening? A case series. BMJ Case Rep 2015; 2015: bcr2014207234.

26. Bohnhorst B. Usefulness of abdominal ultrasound in diagnosing necrotizing enterocolitis. Arch Dis Fetal Neonatal Ed 2013; 98: 445-450.

27. Ade-Ajayi N, Veys P, Stanton M, et al. Conservative management of pneumatosis intestinalis and pneumoperitoneum following bone-marrow transplantation. Pediatr Surg Int 2002; 18: 692-695.

28. Al-Talib A, Al-Ghtani F, Munk R. Pneumatosis intestinalis: can we avoid surgical intervention in nonsurgical patients. Case Rep Gastroenterol 2009; 2: 286-292.

29. Umapathi B, Friel C, Stukenborg G, Hedrick T. Estimating the risk of bowel ischemia requiring surgery in patients with tomographic evidence of pneumatosis intestinalis. Am J Surg 2016; 212: 762-768.

30. Kameyarma K, Noguchi Y, Matsumoto T, et al. Pneumatosis intestinalis in a patient of myasthemia gravis treated with high-dose corticosteroid. Rinsho Shikeigaku 2003; 43: 277-280.

31. Nidimusili A, Mennella J, Shaheen K. Small intestinal ischemia with pneumatosis in a young adult: what could be the cause? Case Rep Gastrointest Med 2013; 2013: 462985. 